\title{
Decoherence and microscopic diffusion at the Sachdev-Ye-Kitaev model
}

\author{
Javier M. Magán* \\ Instituto Balseiro, Centro Atómico Bariloche 8400-S.C. de Bariloche, Río Negro, Argentina
}

(Received 12 January 2017; revised manuscript received 26 March 2018; published 10 July 2018)

\begin{abstract}
Sachdev-Ye-Kitaev (SYK) or embedded random ensembles are models of $N$ fermions with random k-body interactions. They play an important role in understanding black hole dynamics, quantum chaos, and thermalization. We study out-of-equilibrium scenarios in these systems and show how they display perfect decoherence at all times. This peculiar feature makes them very attractive in the context of the quantum-toclassical transition and the emergence of classical general relativity. Based on this feature and unitarity, we propose a rate/continuity equation for the dynamics of the $\mathcal{O}\left(e^{N}\right)$ microstates probabilities. The effective permutation symmetry of the models drastically reduces the number of variables, allowing for compact expressions of n-point correlation functions and entropy of the microscopic distribution. Further assuming a generalized Fermi golden rule allows finding analytic formulas for the kernel spectrum at finite $N$, providing a series of short- and long-time scales controlling the out-of-equilibrium dynamics of this model.
\end{abstract}

DOI: 10.1103/PhysRevD.98.026015

\section{BLACK HOLES, DECOHERENCE AND DETAILED BALANCE}

Finding Planckian corrections to black hole dynamics requires finding finite-size corrections to thermalization processes, since the coupling $\lambda \sim\left(G M^{2}\right)^{-1}$, with $G$ Newton's constant and $M$ the black hole mass, is inversely proportional to black hole entropy $S_{\mathrm{BH}} \sim 1 / \lambda$. But finding finite-size corrections to interacting many-particle states is an almost hopeless task, both analytically and numerically $[1,2]$, a problem with various unwanted consequences. First, verifying unitarity, as predicted by AdS/CFT [3], by finding such corrections is bound to be a very difficult problem $[4,5]$. Second, this obstructs the exploration of time scales diverging with the entropy of the system (much larger than quasinormal relaxation [6]). Finally, when studying out-of-equilibrium processes, one is generically bound to study near-equilibrium scenarios, in which correlation functions of equal-time observables in the evolving state can be related to Green functions at the stationary distribution (linear response theory). Studying out-of-equilibrium processes from a microscopic quantum state perspective is important for both black hole physics (collapse scenarios) and for the study of general aspects of thermalization (departures of linear response theory).

*javier.magan@cab.cnea.gov.ar

Published by the American Physical Society under the terms of the Creative Commons Attribution 4.0 International license. Further distribution of this work must maintain attribution to the author(s) and the published article's title, journal citation, and DOI. Funded by SCOAP.
In this article, we propose and explore one physical feature of black holes, namely dephasing/decoherence of the wave function, which has the potential to overcome part of these problems. In chaotic systems, unitary evolution effectively drives the state towards a stationary diagonal state, given by the microcanonical probabilities of each microstate. This aspect can be easily checked numerically in systems with few degrees of freedom, and it can be generically argued by assuming the usual random state approximation [7]. On the road towards stationarity, we expect the appearance of three important time scales: (i) A decoherence time scale $t_{\mathrm{d}}$, the time at which reduced states are specified by its diagonal entries (the probabilities), (ii) a Markovian time scale $t_{\mathrm{M}}$, the time at which the probabilities satisfy a Markovian equation, and (iii) a global relaxation time scale $t_{\mathrm{r}}$, the time at which all diagonal elements saturate to their stationary values. It is plain that for any system $t_{\mathrm{d}} \leq t_{\mathrm{M}} \leq t_{\mathrm{r}}$. The first inequality expresses that, for times smaller than $t_{\mathrm{M}}$, the state, although effectively described by its diagonal, still contains important information in the off-diagonal entries if we seek to compute the time evolution. The second inequality notices that for times greater than $t_{\mathrm{r}}$, the probabilities satisfy trivially the rate equation (see below). But the hierarchies between the three time-scales might change from one system to another, and interesting simplifications might occur whenever $t_{\mathrm{d}} \leq t_{\mathrm{M}} \ll t_{\mathrm{r}}$.

Since black holes are expected to saturate the bound on chaos [8], we expect decoherence to appear in its strongest form and that the hierarchy $t_{\mathrm{d}} \ll t_{\mathrm{r}}$ indeed takes place. To show this is the case, we will use Sachdev-Ye-Kitaev (SYK)-type models $[9,10]$,

$$
H=\sum_{\substack{1 \leq i_{1}<\ldots<i_{k} \leq N \\ 1 \leq j_{1}<\ldots<j_{k} \leq N}} J_{i_{1} \ldots i_{k} ; j_{1} \ldots j_{k}} c_{i_{1}}^{\dagger} \ldots c_{i_{k}}^{\dagger} c_{j_{1}} \ldots c_{j_{k}},
$$


where $J_{i_{1} \ldots i_{k} ; j_{1} \ldots j_{k}}$ are real independent random numbers with zero mean and variance equal to $J$, while $c^{\dagger}$ and $c$ create and destroy spinless fermions. In this version, Hermiticity requires $J_{i_{1} \ldots i_{k} ; j_{1} \ldots j_{k}}=J_{j_{1} \ldots j_{k} ; i_{1} \ldots i_{k}}$, each term contains $k$ annihilation and $k$ creation operators, and the number of particles $N_{T}=\sum_{i=1}^{N} c_{i}^{\dagger} c_{i}$ is conserved [11]. An average over the ensemble of $\mathcal{O}$ will be denoted by $\overline{\mathcal{O}}$.

These models were studied for quantum chaos [14]. They were named k-body embedded ensembles and were proposed to improve the random Hamiltonian approximation [15], which assumes interactions for every $k$. Recent interest grew mainly due to Kitaev [9], who argued they have holographic duals and saturate the chaos bound [8], and Sachdev [10], who showed they have the right entropy. A complete discussion in these topics is given in [16], and interesting further aspects were developed in [17-32]. Concurrently, these models were used to discuss aspects of eigenstate thermalization analytically [12,33-35], and a relation between large- $\mathrm{N}$ factorization and entanglement evolution was found [13]. Besides, these models might be constructed in the lab [36]. Finally, this model was used in [37] to advance the program started by Maldacena in Ref. [38], followed by [39-43], concerning the quasiperiodicity of correlation functions in finite entropic systems.

We will work in Fock space, in the subspace of $m$ excited particles. The basis $|i\rangle$ contains $i=1, \ldots,\left(\begin{array}{c}N \\ m\end{array}\right)$ states, corresponding to all possible ways of locating the $m$ particles. Starting in one basis state $|i\rangle$, the global state at time $t$ reads

$\rho(t)=\sum_{j, j^{\prime}}\langle j|U(t)| i\rangle\left\langle i\left|U^{\dagger}(t)\right| j^{\prime}\right\rangle|j\rangle\left\langle j^{\prime}\left|\equiv \sum_{j, j^{\prime}} \rho_{j j^{\prime}}(t)\right| j\right\rangle\left\langle j^{\prime}\right|$.

To study decoherence in the evolution, let us first define $\left\langle j\left|H^{n}\right| i\right\rangle \equiv\left(H^{n}\right)_{j i}$, and prove that

$\overline{\left(H^{n}\right)_{j i}\left(H^{m}\right)_{i j^{\prime}}}=\sum_{k_{i}} \overline{H_{j k_{1}} H_{k_{1} k_{2}} \ldots H_{k_{n-1} i} H_{i k_{n}} \ldots H_{k_{n+m-2} j^{\prime}}} \propto \delta_{j j^{\prime}}$.

To understand the intuition, let us work out the two simplest cases. We begin with $n=m=1$, which is explicitly computed in the next section. For the Hamiltonian (1), a matrix element $H_{i j}$ is (if nonzero) the sum over $a$ of the random couplings $J_{i j}^{a}$ that take us from state $i$ to $j$, where the index $a$ runs over all such possible "paths." The previous average for $n=m=1$ can thus be written as

$$
\overline{(H)_{j i}(H)_{i j^{\prime}}}=\overline{\left(\sum_{a} J_{i j}^{a}\right)\left(\sum_{a} J_{i j^{\prime}}^{a}\right)} .
$$

The crucial observation is that if $j \neq j^{\prime}$, all such couplings are associated to different terms of the Hamiltonian (1).
They are therefore independent random numbers and the average is zero. On the other hand, due to Hermiticity of the Hamiltonian, in the case $j=j^{\prime}$, the two sums over paths (the sums over $a$ ) run exactly over the same set of random couplings $J$, and when taking the average, each term $J_{i j}^{a}$ in the first sum has an equal partner in the second sum. This gives a nonzero average and verifies (3). Intuitively, if for the simplest case $n=m=1$ (a product of two Hamiltonians), we already obtained the desired result, we expect that including products involving more random Hamiltonians could only make things worst, and certainly do not destroy the $\delta_{j j^{\prime}}$ feature.

Let's prove the following nontrivial case, given by $n=m=2$. The average reads

$$
\begin{aligned}
& \overline{\left(H^{2}\right)_{j i}\left(H^{2}\right)_{i j^{\prime}}} \\
& =\sum_{k_{1} k_{2}} \overline{H_{j k_{1}} H_{k_{1} i} H_{i k_{2}} H_{k_{2} j^{\prime}}} \\
& =\sum_{k_{1} k_{2}} \overline{\left(\sum_{a} J_{j k_{1}}^{a}\right)\left(\sum_{a} J_{k_{1} i}^{a}\right)\left(\sum_{a} J_{i k_{2}}^{a}\right)\left(\sum_{a} J_{k_{2} j^{\prime}}^{a}\right) .}
\end{aligned}
$$

Since the random couplings have Gaussian statistics, such an average is the sum over all possible pairings between the different parentheses. By using the previous $n=m=1$ case, two such pairings are trivially seen to produce $\delta_{j j^{\prime}}$. The problematic case is the following term:

$$
\overline{\left(\sum_{a} J_{j k_{1}}^{a}\right)\left(\sum_{a} J_{i k_{2}}^{a}\right)} \overline{\left(\sum_{a} J_{k_{1} i}^{a}\right)\left(\sum_{a} J_{k_{2} j^{\prime}}^{a}\right)} .
$$

This is tricky because the two separate averages need not be zero even in the case in which $j k_{1} \neq i k_{2}$. This happens when the very same Hamiltonian terms that take us from $j$ to $k_{1}$ take us also from $i$ to $k_{2}$. In any case, it can be seen that the product of the two averages is proportional to $\delta_{j j^{\prime}}$. To observe this feature, we need to come back and construct the chain of operators associated to the transitions, but now with the knowledge of the specific contractions we have made. If we used the term $\hat{H}_{j k_{1}}^{a}$, associated to a path $a$ from $j$ to $k_{1}$ with coupling $J_{j k_{1}}^{a}$, then the opposite transition $\hat{H}_{k_{1} j}^{a}$ (with the same coupling due to Hermiticity), has to occur somewhere in the string. This is due to the fact that we have contracted $J_{j k_{1}}^{a}$ with $J_{i k_{2}}^{a}$, and that both transitions are associated to the same type of terms in the Hamiltonian. Notice that the forward transition gives zero answer. The same happens with the term $\hat{H}_{i k_{2}}^{a}$, due to the other contraction. In such an "unordered" string of operators, every path we take has an inverse path:

$$
\hat{H}_{j k_{1}}^{a} \hat{H}_{i k_{2}}^{a} \hat{H}_{k_{1} j}^{a} \hat{H}_{k_{2} i}^{a} \text {. }
$$

Therefore, applying such a string of operators to a given state $\left|j^{\prime}\right\rangle$ provides $\left|j^{\prime}\right\rangle$ as an outpout (or zero which does not 
contribute), so that the only way that (6) can be nonzero is if $j=j^{\prime}$.

The same argument is valid for the general case. The right-hand side of (3) can be written as

$$
\begin{aligned}
& \overline{\left(H^{n}\right)_{j i}\left(H^{m}\right)_{i j^{\prime}}} \\
& =\sum_{k_{i}} \overline{\left(\sum_{a} J_{j k_{1}}^{a}\right)\left(\sum_{a} J_{k_{1} k_{2}}^{a}\right) \ldots\left(\sum_{a} J_{k_{n+m-2} j^{\prime}}^{a}\right) .}
\end{aligned}
$$

Again, such an average is the sum over all possible pairings. For such pairings we can again construct the chain of operators associated to each transition, taking into account the contractions we have made. Such pairings imply that if we used one term, such as $\hat{H}_{k_{1} k_{2}}^{a}$, associated to a path $a$ from $k_{1}$ to $k_{2}$ with coupling $J_{k_{1} k_{2}}^{a}$, then the opposite transition $\hat{H}_{k_{2} k_{1}}^{a}$ (with the same coupling due to Hermiticity) has to occur somewhere in the string. Again, in such an "unordered" string of operators, every path we take has an inverse path somewhere in the string. Applying such a string of operators to $\left|j^{\prime}\right\rangle$ provides $\left|j^{\prime}\right\rangle$ as an output, and we conclude that the only way (6) can be nonzero is if $j=j^{\prime}$.

Since by expanding the unitary evolution in (2), all terms are of the form (3), we arrive at the following important conclusion:

$$
\overline{\rho(t)}=\sum_{j} \overline{|\langle j|U(t)| i\rangle|^{2}}|j\rangle\left\langle j\left|\equiv \sum_{j} p_{j}(t)\right| j\right\rangle\langle j| .
$$

For exactly the same reasons, dividing the system in $A$ and $B$ (any possible division), with Fock basis $j_{A}$ and $j_{B}$, provides diagonal reduced states on average:

$$
\begin{aligned}
& \overline{\rho_{A}(t)}=\overline{\operatorname{Tr}_{B} \rho(t)}=\sum_{j_{A}} p_{j_{A}}(t)\left|j_{A}\right\rangle\left\langle j_{A}\right|=\operatorname{Tr}_{B} \overline{\rho(t)} \\
& \overline{\rho_{B}(t)}=\overline{\operatorname{Tr}_{A} \rho(t)}=\sum_{j_{B}} p_{j_{B}}(t)\left|j_{B}\right\rangle\left\langle j_{B}\right|=\operatorname{Tr}_{A} \overline{\rho(t) .}
\end{aligned}
$$

We recognize an extremely peculiar feature of these models: starting at any fixed $|i\rangle$, the state is fully specified on average by its diagonal at any time. Global dephasing and local decoherence are as strong as they can be, the decoherence time being zero. One of the hierarchies is thus satisfied $t_{\mathrm{d}} \ll t_{\mathrm{r}}$. Notice that the same would happen in the generalized SYK models with spatial dimensions studied in [23]. This type of decoherence has been shown to generalize to large $\mathrm{N}$ vector models and gauge theories in [44].

Now, since off-diagonal entries are complex random numbers which vanish on average at all times, the 'probability current' $p_{j}(t)$ is expected to satisfy a microscopic continuity equation,

$$
\frac{d p_{j}(t)}{d t}=\sum_{i} \Gamma_{i \rightarrow j}(t) p_{i}(t)-\left(\sum_{i} \Gamma_{j \rightarrow i}(t)\right) p_{j}(t),
$$

where $\Gamma_{i \rightarrow j}(t)$ are transition probability rates between microstates $i$ and $j$. We assume this is the case, i.e., that the Markovian time scale is small as well, as it is assumed in other decoherence scenarios associated with different conserved currents. Indeed, such behavior can be seen to hold at sufficiently small times, i.e., for the first term in the expansion of the unitary evolution. But in this case, we could not give a full proof, valid at any time scale, as with decoherence.

Unitarity implies the detailed balance condition,

$$
\sum_{i} \Gamma_{i \rightarrow j}(t)=\sum_{i} \Gamma_{j \rightarrow i}(t) \equiv \Gamma(t),
$$

expressing the potential of unitarity to relate lifetimes to more complicated observables. Equation (10) is a novel perspective on large- $\mathrm{N}$ classicalization, containing $1 / N$ effects and long-time scales.

In particular, one of the main outputs of our analysis is that the time scale in which such a process reaches stationarity diverges with the entropy of the system as $t_{\mathrm{r}} \sim \mathcal{O}(S)$. In black holes, such time scales appear in two different and, in principle, unrelated scenarios. The first one is that it is the time scale in which the black hole loses a $\mathcal{O}(1)$ part of its mass. This is because a black hole emits one particle with energy of order $T$ in a time of order $\beta$. The second reason is more related to our results. The time scale $t_{\mathrm{r}} \sim \mathcal{O}(S)$ is the time scale at which two-point correlation functions, which decay exponentially to zero in the semiclassical limit, have to stop decaying and should reach stationarity at a value of $\mathcal{O}\left(e^{-S}\right)$. This is the so-called quantum chaotic regime and was extensively discussed in [38-43], where it is argued on general unitarity grounds. In our approach, such a saturation at such a time scale will appear dynamically, as the saturation of the previous Markov process. We will see that the time $t_{\mathrm{r}} \sim \mathcal{O}(S)$ is both the time at which the probability reaches stationarity and the time at which correlation functions reach stationarity.

One remark is needed. Notice that (9) transparently shows how the averaging procedure implies certain information loss and a natural coarse-graining (a usually unnoticed fact). It might seem strange we can recover $1 / N$ effects after taking the average. Related to the previous paragraph, a very nontrivial $1 / N$ effect naturally taken into account by Eqs. (9) and (10) is the following: Unitarity (in the form of detailed balance) ensures that the process drives an initial distribution towards the stationary state $p_{i}^{h}=1 /\left(\begin{array}{l}N \\ m\end{array}\right) \sim \mathcal{O}\left(e^{-N}\right)$, a strongly nonperturbative effect. This effect, missed in any gravitational description, was used in [38] to discuss information loss in AdS/CFT. To our knowledge, there are no dynamical computations obtaining these nonperturbative effects, only generic arguments involving unitarity. Within the present framework, probabilities and correlation functions tend to nonzero values in a dynamical fashion. Given that at long times we reproduce these nonperturbative effects, it is natural to expect we recover other $1 / N$ effects during evolution. At any rate, we remark that such a result is the full answer on average, as proven by (3). 


\section{EXACT CONSEQUENCES OF PERMUTATION SYMMETRY}

SYK displays an effective permutation symmetry on average, the relabeling of the fermions. In what follows, states differ from each other by moving a number $\alpha$ of particles are said to be distance $\alpha$ apart.

Say $\left|\psi_{\text {in }}\right\rangle=|i\rangle$ (a given Fock basis state), the most important consequence of permutation symmetry is that the evolution of probabilities $p_{j}^{\alpha i}(t)$ of states $|j\rangle$ that are $\alpha$ particles apart from $|i\rangle$ is the same in all of them:

$$
\left|\psi_{\text {in }}\right\rangle=|i\rangle \Rightarrow p_{j}^{\alpha i}(t)=p_{k}^{\alpha i}(t) .
$$

We only need to compute $p_{\alpha} \equiv p_{j}^{\alpha i}$ for $\alpha=0, \ldots, m$, with $p_{\alpha=0} \equiv p_{i}$. This is similar to thermodynamics, in which probabilities only depend on macroscopic quantities, such as energy. Here, the classical macroscopic variable is the number of particles which have been moved. Each such sector contains a huge number of indistinguishable microstates, corresponding to all possible ways of choosing and moving $\alpha$ particles, all with the same probability. Joining this observation with the previously derived decoherence, the state can thus be written as a sum over classically distinct configurations as

$$
\overline{\rho(t)}=\sum_{\alpha=0}^{m}\left(\begin{array}{c}
m \\
\alpha
\end{array}\right)\left(\begin{array}{c}
N-m \\
\alpha
\end{array}\right) p_{\alpha}(t)\left(\prod_{i_{\alpha}}\left|i_{\alpha}\right\rangle\left\langle i_{\alpha}\right|\right) .
$$

Permutation symmetry reduces a seemingly exponentially hard problem of $\left(\begin{array}{c}N \\ m\end{array}\right)$ variables to a polynomially hard problem with $m+1 \leq N / 2+1$ variables. This is an explicit example of the generic results described in [44] concerning the state structure on large- $\mathrm{N}$ theories.

The task is to map (10) to $m+1$ coupled differential equations involving $p_{\alpha}$. To do so, notice the second consequence of permutation symmetry. The transitions rates $\Gamma_{i \rightarrow j}(t) \equiv \Gamma_{d}$ only depend on the distance $d$ between the states $i$ and $j$, and not on the states themselves.

Given these comments, the following result is exact, provided (10) holds. The probability $p_{\alpha}(t)$ of states $\alpha$ particles away from $|i\rangle$, with $\left|\psi_{\text {in }}\right\rangle=|i\rangle$, satisfies

$$
\frac{d p_{\alpha}}{d t}=-\Gamma(t) p_{\alpha}(t)+\sum_{\beta=0}^{m} C_{\alpha}^{\beta}(t) p_{\beta}(t),
$$

where

$$
\begin{aligned}
C_{\alpha}^{\beta}= & \sum_{j=0}^{2 \alpha} \Gamma_{\beta-\alpha+j}(t) \\
& \times \sum_{l=0}^{j}\left(\begin{array}{c}
\alpha \\
j-l
\end{array}\right)\left(\begin{array}{c}
\alpha \\
l
\end{array}\right)\left(\begin{array}{c}
N-m-\alpha \\
\beta-\alpha+j-l
\end{array}\right)\left(\begin{array}{c}
m-\alpha \\
\beta-\alpha+l
\end{array}\right) .
\end{aligned}
$$

The derivation was a case-by-case study. Here we explain its logic directly. The sum over $\beta$ adds contributions from state $\beta$ particles separated from $i$ to state $\alpha$ particles separated from $i$. We need to consider all distances $\beta$, explaining the sum in (14). For each $\beta$, we need to examine all possible distances $d$ from $\beta$ to $\alpha$. For distance $d$, the contribution to $\alpha$ reads

$$
\Gamma_{d}(t) \Omega_{d},
$$

where $\Omega_{d}$ is the number of $\beta$ states $d$ away from $\alpha$, a number we need to compute. First, if the distance is not within $\beta-\alpha \leq d \leq \beta+\alpha$, the number of such contributions is zero. The reason is as follows. Suppose that the $\alpha$ particles and $\alpha$ holes that differ from $|i\rangle$ in a state $\alpha$ are all located in different places than the $\beta$ particles and $\beta$ holes that differ from $|i\rangle$ in a state $\beta$. The distance between such states is $\beta+\alpha$, the maximum one. The minimum distance occurs when the $\alpha$ particles and $\alpha$ holes that differ from $|i\rangle$ in a state $\alpha$ are all contained in the $\beta$ particles and $\beta$ holes that differ from $|i\rangle$ in a state $\beta$. Here, the distance is $\beta-\alpha$, the minimum one. Summing over $d$ is summing from $j=0$ to $j=2 \alpha$ with $d_{j}=\beta-\alpha+j$, explaining the first sum in (15), where one should notice that $2 \alpha-j$ is the number of particles plus holes shared by states $\alpha$ and $\beta$ but not shared with $|i\rangle$. This $2 \alpha-j$ sharing can occur in various ways. They can share $\alpha$ particles and $\alpha-j$ holes, or $\alpha-1$ particles and $\alpha-j+1$ holes...or $\alpha-j-l$ particles and $\alpha-$ $j+l$ holes... until sharing $\alpha-j$ particles and $\alpha$ holes. The number of each possibility is a straightforward balls and bins problem which justifies the last sum in $l$ in Eq. (15).

Equation (14) shows the emergence of a local onedimensional diffusion-type equation from SYK, a model which at first sight shows no such structure. The label $k$, which enters the definition of the model as the number of fermions participating in the interaction, controls the amount of nonlocality in the diffusion process.

The next results concerns entropy and correlation functions. They do not depend on the Markovian approximation. They are exact on average given (9). Such an equation implies that the observables of direct interest are of the form

$$
\mathcal{O}=\sum_{k} \mathcal{O}_{k}|k\rangle\langle k|,
$$

where $|k\rangle$ is the Fock basis defined in the previous section. Any string as

$$
\mathcal{O}_{i j \ldots l}=n_{i} n_{j} \ldots n_{l},
$$

where $n_{i} \equiv c_{i}^{\dagger} c_{i}$, belongs to the diagonal algebra (17). Taking again $|i\rangle$, with the first $m$ particles excited as the initial state, and setting $j \leq m$ and $j^{\prime} \leq N-m$, the generic $n$-point correlation function is

$$
\begin{aligned}
& \operatorname{Tr}\left(\overline{\rho(t)} n_{i} \ldots n_{j} n_{m+1} \ldots n_{m+j^{\prime}}\right) \\
& \quad=\sum_{\alpha=j^{\prime}}^{m-j}\left(\begin{array}{c}
m-j \\
\alpha
\end{array}\right)\left(\begin{array}{c}
N-m-j^{\prime} \\
\alpha-j^{\prime}
\end{array}\right) p_{\alpha}(t) .
\end{aligned}
$$


The product of binomials is the number of states at distance $\alpha$ giving nonzero contribution (equal to one). Thus computing $p_{\alpha}(t)$ allows finding all $\mathrm{n}$-point correlations, without worrying about the number of insertions.

It is obvious from the previous expression that by the time the probability distribution reaches stationarity, the correlation functions reach stationarity as well, and vice versa. It is generically believed that correlation functions stop decaying at a time of order $\mathcal{O}(S)$, see [38-43]. The arguments given for that saturation are always very general, based on quasiperiodicity and unitarity. Below we show how such a time scale appears dynamically in our framework, due to detailed balance (11).

We remark that although in experimental setups equaltime correlators of the previous type are not easy to measure, ${ }^{1}$ when the process reaches near equilibrium such equal-time correlators are related to Green functions at finite temperature, and can thus be used to obtain measurable quantities and finite departures from the linear approximation.

Finally, entanglement entropy of the diagonal algebra reads

$$
\begin{aligned}
S\left(\rho_{D}\right) & =-\sum_{k=1}^{\left(\begin{array}{c}
N \\
m
\end{array}\right)} p_{k}(t) \log p_{k}(t) \\
& =-\sum_{\alpha=0}^{m}\left(\begin{array}{c}
m \\
\alpha
\end{array}\right)\left(\begin{array}{c}
N-m \\
\alpha
\end{array}\right) p_{\alpha}(t) \log p_{\alpha}(t) .
\end{aligned}
$$

This is a sum over the classically distinct subsectors defined by parameter $\alpha$.

Given that the saturation of this entropy implies saturation of all operators belonging to the diagonal algebra, one is tempted to conclude it relates to complexity evolution [45-51]. Care has to be taken since, as we show below, this entropy saturates at a time of order $t \sim \mathcal{O}(N)$, much faster than expected for complexity.

\section{ONE-DIMENSIONAL K-LOCAL DIFFUSION}

The probabilities $p_{\alpha}(t)$ are, on average, a convenient and sufficient set. To proceed, we need an expression for the transition rates. In this section, we develop two approximations of increasing strength. First, as in other continuity equations, we can assume the transition rates to be nonzero only between states directly connected by the Hamiltonian [52]. This natural "locality" assumption, expected to be accurate in the thermodynamic limit, results in the following k-local diffusion equation,

$$
\frac{d p_{\alpha}}{d t}=-\Gamma(t) p_{\alpha}+\sum_{\beta=\alpha-k}^{\alpha+k} C_{\alpha}^{\beta}(t) p_{\beta}
$$

\footnotetext{
${ }^{1}$ Research in quantum tomography is advancing in such direction.
}

where $C_{\alpha}^{\beta}(t)$ is defined as above. In this approximation, we only need to measure/compute $\Gamma_{1}, \ldots \Gamma_{k}$, two functions for the physical $k=2$ case with two-body interactions. Then (21) together with the results of the previous section would provide the correlation functions for the nonequilibrium process.

A stronger approximation is the following:

$$
\Gamma_{i \rightarrow j}(t)=D(t) \overline{|\langle j|H| i\rangle|^{2}}
$$

for some function $D(t)$. Notice that at small times $D(t) \propto t$ for any Hamiltonian, while Fermi golden rule $D(t)=D$ is expected to hold after some time and last for longer times, given that in the thermodynamic limit each basis state decays to a continuum in SYK. These regimes have been observed in SYK models [2]. The Hamiltonian matrix element between $|i\rangle$ and $\left|j_{\alpha}\right\rangle$ separated by distance $\alpha$ is:

$\overline{\left|\left\langle j_{\alpha}|H| i\right\rangle\right|^{2}}=\overline{\left(\sum_{\text {paths }} J_{\text {paths }}\right)^{2}}=\Omega_{\text {paths }} J^{2}=\left(\begin{array}{c}m-\alpha \\ k-\alpha\end{array}\right) J^{2}$,

since after moving $\alpha$ particles we have $\left(\begin{array}{c}m-\alpha \\ k-\alpha\end{array}\right)$ 'paths' to create and destroy fermions without affecting the final state. Since each path contributes a random number, averaging the paths sum squared gives the number of paths times the variance of one path. With this approximation we arrive at:

$$
\frac{d p_{\alpha}}{d t}=-\Gamma(t) p_{\alpha}+D(t) \sum_{\beta=\alpha-k}^{\alpha+k} C_{\alpha}^{\beta} p_{\beta},
$$

where:

$$
\begin{aligned}
C_{\alpha}^{\beta}= & \sum_{j=0}^{2 \alpha}\left(\begin{array}{c}
m-(\beta-\alpha+j) \\
k-(\beta-\alpha+j)
\end{array}\right) \\
& \times \sum_{l=0}^{j}\left(\begin{array}{c}
\alpha \\
j-l
\end{array}\right)\left(\begin{array}{c}
\alpha \\
l
\end{array}\right)\left(\begin{array}{c}
N-m-\alpha \\
\beta-\alpha+j-l
\end{array}\right)\left(\begin{array}{c}
m-\alpha \\
\beta-\alpha+l
\end{array}\right) .
\end{aligned}
$$

The relation between $\Gamma(t)$ and $D(t)$ is fixed by the detailed balance condition (11), as usual in Markov processes. Such a condition can be more transparently imposed in the case of $p_{0}$, which is the return probability to the initial state. The equation for $p_{0}$ could have been inferred without the complicated technical derivation of the previous section. It is just given by

$$
\frac{d p_{0}}{d t}=-\Gamma(t) p_{0}+D(t) \sum_{\beta=0}^{k} C_{0}^{\beta} p_{\beta}
$$

where

$$
C_{0}^{\beta}=\left(\begin{array}{c}
m-\beta \\
k-\beta
\end{array}\right)\left(\begin{array}{c}
N-m \\
\beta
\end{array}\right)\left(\begin{array}{c}
m \\
\beta
\end{array}\right)
$$


This equation just says that the time evolution of $p_{0}$ is minus its decay rate plus the probability of return transitions, which for $p_{0}$ is simply given by the above expression (the rate times the number of possible transitions associated to that rate). Detailed balance fixes then the relation between $\Gamma(t)$ and $D(t)$ to be

$$
\Gamma(t)=D(t) \sum_{\beta=0}^{k}\left(\begin{array}{c}
m-\beta \\
k-\beta
\end{array}\right)\left(\begin{array}{c}
m \\
\beta
\end{array}\right)\left(\begin{array}{c}
N-m \\
\beta
\end{array}\right),
$$

so that in the present framework, there is only one unknown function, which is the decay rate of the initial state.

A very nontrivial consistency check of the complete coupled system of rate equations, as defined by (24) and (25), is that once we have imposed the detailed balance condition by using the equation for $p_{0}(t)$, and derive the previous relation between $\Gamma(t)$ and $D(t)$, such a relation should be respected by all the other coupled equations for different $\alpha$. This can actually be verified by plugging in the expression (25) in MATHEMATICA, whose output is that, independent of $\alpha$, the sum over $\beta$ respects the detailed balance condition:

$$
\sum_{\beta=\alpha-k}^{\alpha+k} C_{\alpha}^{\beta}=\sum_{\beta=0}^{k}\left(\begin{array}{c}
m-\beta \\
k-\beta
\end{array}\right)\left(\begin{array}{c}
m \\
\beta
\end{array}\right)\left(\begin{array}{c}
N-m \\
\beta
\end{array}\right)=\frac{\Gamma(t)}{D(t)} .
$$

The interesting aspect of this approximation is that, up to one function $\Gamma(t)$, which might be obtained also by computing the two-point correlation function, the manybody system can almost be solved exactly, obtaining nontrivial aspects of the long time dynamics, as we show in the next section.

\section{KERNEL SPECTRUM AND TIME SCALES}

The diffusion process can be written as

$$
\frac{d p}{d t}=\Gamma(t) M p(t),
$$

where the $(m+1) \times(m+1)$ matrix $M$ is defined to match Eqs. (24) and (25). The solution is

$$
p(t)=\sum_{a=1}^{m+1} c_{a} e^{\lambda_{a} \int_{0}^{t} \Gamma\left(t^{\prime}\right) d t^{\prime}} v^{a},
$$

where $\lambda_{a}$ and $v^{a}$ are the $m+1$ eigenvalues and eigenvectors of $M$, and the $c_{a}$ are fixed by the initial condition $p_{\alpha}(t)=\delta_{\alpha, 0}$. As usual, $c_{1} v_{1}=1 /\left(\begin{array}{c}N \\ m\end{array}\right)$ and $\lambda_{1}=0$, corresponding to the stationary distribution. In what follows, we set $N=2 m$.

The equation can be solved by numerical methods. When doing so we found something striking and unexpected. The kernel spectrum is composed by series of fractional numbers following specific laws [56]. We could not prove analytically the following relations, but the reader can verify them for any $N=2 \mathrm{~m}$. The kernel spectrum $\lambda_{i}^{k}(m)$, organized as $\lambda_{1}^{k}(m)=0>\lambda_{2}^{k}(m) \geq \ldots \geq \lambda_{m+1}^{k}(m)=$ -1 , reads:

$$
\lambda_{i}^{k}(m)=\frac{(i-1)(i-2(1+m))\left(\sum_{q=0}^{2 k-2} P(m, q) i^{q}\right)}{(m-k+1) \cdots(m-1) m(m+1) \cdots(m+k)},
$$

where $P(m, 2 k-2)=1 \quad$ and $\quad P(m, 0)=k(m-k+2) \ldots$ $m(m+2) \ldots(m+k)$. For $k=1,2$

$$
\begin{aligned}
\lambda_{i}^{1}(m)= & \frac{(i-1)(i-2(1+m))}{m(m+1)} \\
\lambda_{i}^{2}(m)= & \frac{(i-1)(i-2(1+m))}{(m-1) m(m+1)(m+2)} \\
& \times\left(i^{2}-i(3+2 m)+2 m(2+m)\right) .
\end{aligned}
$$

Formulas for $k=3,4,5$ were explicitly computed but a generic expression as a function of $k$ was not found.

Equations (29) and (30) are the last main results of the article. They provide $m$ time scales which might be measured in experiments [36] (more precisely one can measure all ratios). It is remarkable that such a formula for the $i$ eigenvalue even exists, suggesting the influence of some hidden symmetry [57].

As a direct application, since the second eigenvalue (the gap) controls the late time approach to equilibrium:

$$
p_{\alpha}\left(t \gg t_{\mathrm{r}}\right)=\frac{1}{\left(\begin{array}{c}
N \\
m
\end{array}\right)}+c_{2} e^{\lambda_{2}^{k}(m)} \int_{0}^{t} \Gamma\left(t^{\prime}\right) d t^{\prime} v_{\alpha}^{2},
$$

by using the full kernel we find an exact expression for the gap:

$c_{2}(m)=\frac{1-2 m}{\left(\begin{array}{l}N \\ m\end{array}\right)} \quad \lambda_{2}^{k}(m)=-\frac{2 k}{m+k} \quad v_{\alpha}^{2}=\frac{-m+2 \alpha}{m}$.

For $k \sim \mathcal{O}(1)$ we see the natural appearance of time scales of $\mathcal{O}(N)$ (in units of $\tau=\int_{0}^{t} \Gamma\left(t^{\prime}\right) d t^{\prime}$ ) at the edge of the spectrum. These are the longest time scales controlling the out-of-equilibrium process. In particular, from the previous gap relations, we conclude that the global relaxation time $t_{\mathrm{r}}$ described in the introduction, which was defined as the time where probabilities saturate to their stationary value, is of the order of $t_{\mathrm{r}} / \Gamma \sim \mathcal{O}(N)$.

We can now combine this result with the expression for the correlators (19) and conclude that at long times all correlation functions decay with the same exponent, albeit with different prefactors and to different values. Besides, this result verifies in an explicit example the general arguments given in [38-43], concerning unitarity and quasiperiodicity of correlation functions, since it explains in a dynamical fashion why correlation functions saturate to 
their stationary nonpertubative values of order $\mathcal{O}\left(e^{-S}\right)$ on a time scale of $\mathcal{O}(S)$. In our framework, we study a rate equation of the microscopic probability distribution of all basis microstates. Stationarity at the nonperturbative value $p=1 /\left(\begin{array}{l}N \\ m\end{array}\right) \sim \mathcal{O}\left(e^{-N}\right)$ of the probability distribution is ensured by the detailed balance condition (11). Such nonperturbative stationarity, together with the relation between the probabilities and correlation functions (19), implies that the global relaxation time derived here $t_{\mathrm{r}} / \Gamma \sim$ $\mathcal{O}(N)$ controls the saturation of all correlation functions to their nonperturbative values, and this is the expected time scale for the entrance to the so-called quantum chaotic regime.

\section{CONCLUSIONS}

Black hole dynamics is expected to be maximally chaotic [8]. As such, we expect such systems to display the strongest forms of decoherence. This is an interesting aspect of black hole dynamics which has not be considered in the literature.

In this article, we explored SYK, dual to quantum gravity in AdS2 [9,16], in nonequilibrium scenarios from this perspective. We showed that on average, the state (and reduced states) displays exact dephasing/decoherence at all times, since off-diagonal terms vanish (9). Given such feature, and reminding that decoherence roots classical diffusion/continuity equations for conserved currents, we analyzed the generic rate equation (10) for the conserved current at hand, formed by the average microscopic probabilities of the Fock space basis. Such equation interconnects all equal-time correlation functions of the theory. We then analyzed aspects of symmetry, showing how the imply the emergence of a one-dimensional diffusion equation, allowing to extract novel aspects of the long time dynamics.

The main result is a dynamical derivation of the time scale associated to the so-called quantum chaotic regime [38-43], which is usually argued on generic grounds, by using unitarity and the fact that in finite entropy systems two-point correlation functions are quasiperiodic. In our framework, all correlation functions decay to their nonperturbative values of $\mathcal{O}\left(e^{-S}\right)$, and not to zero, and they do so in the expected long time scale, given here by the saturation of the microscopic decay rate process, which we showed to be of $\mathcal{O}(S)$.

We remark that this is the first analytic study of a chaotic system in a far from equilibrium scenario from a Hilbert space perspective. In particular, all recent SYK studies concern correlation functions in the thermal ensemble, which are related to equal-time correlation functions when the process is near equilibrium. Therefore, our approach might open new possibilities in the study of general departures of linear response theory and of far from equilibrium processes.

Finally, from a parallel perspective, Eqs. (9) and (10) are an interesting new look to large-N classicalization, which generalizes to other microscopic models of black holes, as developed in [44]. From this perspective, the generic lessons are the following. Due to strong decoherence in Fock space in large-N theories, and that microstates decay to a continuum in the thermodynamic limit, the diagonal algebra in Fock space is controlled by a rate equation (like a nuclear chain reaction process) typical of an open system, with the bath being the nondiagonal operator algebra. The entanglement between both algebras is the entropy of the probability distribution, and at long times entanglement saturates to the thermal (black hole) entropy. Such rate equation contains $1 / N$ effects, long time scales, and controls many nontrivial correlation functions, including those with a number of insertions scaling with the total number of degrees of freedom.

\section{ACKNOWLEDGMENTS}

It is a pleasure to thank Eduardo Testé Lino for helping in the derivation of the kernel spectrum. We also wish to thank Horacio Casini for discussions around the diagonal operator algebra, and Juan Maldacena and José Barbón for comments on the draft. This work was supported by the Simons Foundation through the It From Qubit Collaboration.
[1] C. Gogolin and J. Eisert, Equilibration, thermalisation, and the emrgence of statistical mechanics in closed quantum systems, Rep. Prog. Phys. 79, 056001 (2016).

[2] F. Borgonovi, F. M. Izrailev, L. .F. Santos, and V. G. Zelevinsky, Quantum chaos and thermalization in isolated systems of interacting particles, Phys. Rep. 626, 1 (2016).

[3] J. M. Maldacena, The large N limit of superconformal field theories and supergravity, Adv. Theor. Math. Phys. 2, 231 (1998).
[4] V. Balasubramanian and B. Czech, Quantitative approaches to information recovery from black holes, Classical Quantum Gravity 28, 163001 (2011).

[5] D. Harlow and P. Hayden, Computational complexity vs firewalls, J. High Energy Phys. 06 (2013) 085.

[6] E. Berti, V. Cardoso, and A. O. Starinets, Quasinormal modes of black holes and black branes, Classical Quantum Gravity 26, 163001 (2009).

[7] J. M. Magan and S. Vandoren, Entanglement in Fock space of random QFT states, J. High Energy Phys. 07 (2015) 150. 
[8] J. Maldacena, S. H. Shenker, and D. Stanford, A bound on chaos, J. High Energy Phys. 08 (2016) 106.

[9] A. Kitaev, A simple model of quantum holography, in Proceeding of KITP (2015).

[10] S. Sachdev and J. Ye, Gapless Spin Fluid Ground State in a Random, Quantum Heisenberg Ferromagnet, Phys. Rev. Lett. 70, 3339 (1993); S. Sachdev, Bekenstein-Hawking Entropy and Strange Metals, Phys. Rev. X 5, 041025 (2015).

[11] The author already worked [12,13] with this version in which $N_{T}=\sum_{i=1}^{N} c_{i}^{\dagger} c_{i}$ is conserved. We remain in such formulation but techniques apply to the Majorana case. The approach rests on the 'all to all' random interactions and the permutation symmetry it implies.

[12] J. M. Magan, Random Free Fermions: An Analytical Example of Eigenstate Thermalization, Phys. Rev. Lett. 116, 030401 (2016).

[13] J. M. Magan, Black holes as random particles: entanglement evolution in infinite range and matrix models, J. High Energy Phys. 08 (2016) 081.

[14] L. Benet and H. A. Weidenmueller, Review of the k-body embedded ensebmles of gaussian random matrices, J. Phys. A 36, 3569 (2003).

[15] F. Haake, Quantum Signatures of Chaos (Springer, New York, 2010).

[16] J. Maldacena and D. Stanford, Remarks on the SachdevYe-Kitaev model, Phys. Rev. D 94, 106002 (2016).

[17] J. Polchinski and V. Rosenhaus, The spectrum in the Sachdev-Ye-Kitaev model, J. High Energy Phys. 04 (2016) 001.

[18] A. Jevicki, K. Suzuki, and J. Yoon, Bilocal holography in the SYK model, J. High Energy Phys. 07 (2016) 007.

[19] K. Jensen, Chaos in AdS2 Holography, Phys. Rev. Lett. 117, 111601 (2016).

[20] D. Bagrets, A. Altland, and A. Kamenev, Sachdev-YeKitaev model as Liouville quantum mechanics, Nucl. Phys. B911, 191 (2016).

[21] L. Garcia-Alvarez, I. L. Egusquiza, L. Lamata, A. del Campo, J. Sonner, and E. Solano, Digital Quantum Simulation of Minimal AdS/CFT, Phys. Rev. Lett. 119, 040501 (2017).

[22] A. Jevicki and K. Suzuki, Bi-local holography in the SYK model: perturbations, J. High Energy Phys. 11 (2016) 046.

[23] Y. Gu, X. Qi, and D. Stanford, Local criticality, diffusion and chaos in generalized Sachdev-Ye-Kitaev models, J. High Energy Phys. 05 (2017) 125.

[24] D. Gross and V. Rosenhaus, A generalization of SachdevYe-Kitaev, J. High Energy Phys. 02 (2017) 093.

[25] M. Berkooz, P. Narayan, M. Rozalli, and J. Simon, Higher dimensional generalizations of the SYK model, J. High Energy Phys. 01 (2017) 138.

[26] A. M. García -García and J. J. M. Verbaarschot, Spectral and thermodynamics properties of the Sachdev-Ye-Kitaev model, Phys. Rev. D 94, 126010 (2016).

[27] W. Fu, D. Gaiotto, J. Maldacena, and S. Sachdev, Supersymmetric SYK models, Phys. Rev. D 95, 026009 (2017).

[28] E. Witten, An SYK-like model without disorder, arXiv: 1610.09758 .
[29] S. Sachdev and A. A. Patel, Quantum chaos on a critical Fermi surface, in Proc. Natl. Acad. Sci. U.S.A. 114, 1844 (2017).

[30] I. R. Klebanov and G. Tarnopolsky, Uncolored random tensors, melon diagrams, and the SYK models, Phys. Rev. D 95, 046004 (2017).

[31] R. A. Davison, W. Fu, A. Georges, Y. Gu, K. Jensen, and S. Sachdev, Thermoelectric transport in disordered metals without quasiparticles: the SYK models and holography, Phys. Rev. B 95, 155131 (2017).

[32] C. Krishnan, S. Sanyal, and P. N. Balasubramanian, Quantum chaos and holographic tensor models, J. High Energy Phys. 03 (2017) 056.

[33] R. V. Jensen and R. Shankar, Statistical Behavior in Deterministic Quantum Systems with Few Degrees of Freedom, Phys. Rev. Lett. 54, 1879 (1985).

[34] J. M. Deutsch, Quantum statistical mechanics in a closed system, Phys. Rev. A 43, 2046 (1991).

[35] M. Srednicki, Chaos and quantum thermalization, Phys. Rev. E 50, 888 (1994).

[36] I. Danshita, M. Hanada, and M. Tezuka, Creating and probing the Sachdev-Ye-Kitaev model with ultracold gases: Towards experimental studies of quantum gravity, Prog. Theor. Exp. Phys. (2017) 083I01.

[37] J. S. Cotler,G. Gur-Ari, M. Hanada, J. Polchinski, P. Saad, S. Shenker, D. Stanford, A. Streicher, and M. Tezuka, Black holes and random matrices, J. High Energy Phys. 05 (2017) 118.

[38] J. M. Maldacena, Eternal black holes in AdS, J. High Energy Phys. 04 (2003) 021.

[39] J. L. Barbon and E. Rabinovici, Very long time scales and black hole thermal equilibrium, J. High Energy Phys. 11 (2003) 047.

[40] M. Kleban, M. Porrati, and R. Rabadan, Poincare recurrences and topological diversity, J. High Energy Phys. 10 (2004) 030.

[41] V. Balasubramanian, S. Ross, and J. Simon, Black holes, entanglement and random matrices, Classical Quantum Gravity 31, No. 18, 185009 (2014).

[42] J. L. Barbon and E. Rabinovici, Geometry and quantum noise, Fortschr. Phys. 62, 626 (2014).

[43] V. Balasubramanian, B. Craps, B. Czech, and G. Sárosi, Echoes of chaos from string theory black holes, J. High Energy Phys. 03 (2017) 154.

[44] J. M. Magan, De Finetti theorems and entanglement in large-N theories and gravity, Phys. Rev. D 96, 086002 (2017).

[45] A. R. Brown, D. A. Roberts, L. Susskind, B. Swingle, and Y. Zhao, Complexity Equals Action, Phys. Rev. Lett. 116, 191301 (2016).

[46] A. R. Brown, D. A. Roberts, L. Susskind, B. Swingle, and Y. Zhao, Complexity, action, and black holes, Phys. Rev. D 93, 086006 (2016).

[47] J. L. F. Barbón and E. Rabinovici, Holographic complexity and cosmological singularities, J. High Energy Phys. 01 (2016) 084.

[48] J. L. F. Barbón and J. Martin-García, Holographic complexity of cold hyperbolic black holes, J. High Energy Phys. 11 (2015) 181. 
[49] D. A. Roberts and B. Yoshida, Chaos and complexity by design, J. High Energy Phys. 04 (2017) 121.

[50] S. Chapman, H. Marrochio, and R. C. Myers, Complexity of formation in holography, J. High Energy Phys. 01 (2017) 062.

[51] D. Carmi, R. C. Myers, and P. Rath, Comments on holographic complexity, J. High Energy Phys. 03 (2017) 118.

[52] This assumption suggests considering a "Hamiltonian graph." The vertices $V=\left\{|i\rangle ; i=1, \ldots,\left(\begin{array}{c}N \\ m\end{array}\right)\right\}$ are the Fock basis, and the edges are the transition rates $E_{i j}=\Gamma_{d_{i j}}$. A characteristic aspect of SYK is that the graph is regular, homogeneous, isotropic and weighted, with valency $v_{k}=$ $\sum_{\alpha=1}^{k}\left(\begin{array}{c}m \\ \alpha\end{array}\right)\left(\begin{array}{c}N-m \\ \alpha\end{array}\right)$. It is almost an expander graph [53-55], since the diameter is $D \sim \log \Omega$, with $\Omega$ the number of vertices in the graph. The rate equation (21) is a random walk on this graph.

[53] S. Hooy, N. Linial, and A. Wigderson, Expander graphs and their applications, Bull. Am. Math. Soc. 43, 439 (2006).

[54] A. Lubotzky, Expander graphs in pure and applied mathematics, Bull. Am. Math. Soc. 49, 113 (2012).

[55] J. L. F. Barbon and J. M. Magan, Fast scramblers, horizons and expander graphs, J. High Energy Phys. 08 (2012) 016.

[56] We are indebted to Eduardo Testé for collaborating in finding the underlying pattern of the kernel spectrum.

[57] This is further suggested by the structure of eigenvectors, basically equal for all $k$. 